\title{
Internal and external shock fronts in He2-104
}

\author{
M. Contini ${ }^{1,2}$ and L. Formiggini ${ }^{3,4}$ \\ 1 School of Physics \& Astronomy, Tel Aviv University, 69978 Tel Aviv, Israel \\ 2 Astrophysikalisches Institut Potsdam, An der Sternwarte, 16, 14482 Potsdam, Germany \\ 3 Wise Observatory, Tel Aviv University, 69978 Tel Aviv, Israel \\ 4 Istituto di Radioastronomia, CNR, via P. Gobetti 101, 40129 Bologna, Italy \\ Received 21 December 2000 / Accepted 12 June 2001
}

\begin{abstract}
The spectra of both the inner and outer nebulae in He2-104 are modelled by composite models which account consistently for photoionisation and shocks. The temperature of the hot star is found $T_{*} \sim 130000 \mathrm{~K}$. We suggest that the characteristic "crab legs" in He2-104 are produced by R-T instability at the shock front. The calculated X-ray flux is below the detection limit of ROSAT.
\end{abstract}

Key words. shock waves - stars: binaries: symbiotic - stars: individual: He2-104

\section{Introduction}

Symbiotic novae (SBN) are binary systems which consist of a white dwarf (WD) and a late-type giant, often a Mira variable. Colliding winds from both components after an outburst create complex hydrodynamic conditions within the system and outside. The importance of shock hydrodynamics to the evolution of symbiotic systems has been demonstrated by the morphological development at later stages (Hack \& Paresce 1993; Contini 1997; Contini \& Formiggini 1999, etc.). Particularly, new observational techniques detected morphological structures extending up to $2 \mathrm{pc}$, with complex phenomenology (jets, expanding shells, "crab legs", etc.) in the circumstellar material surrounding SBN. Actually, Corradi et al. (1999a) detected an extended optical nebula in 8 D-type (dust rich) SBN, about $40 \%$ of the observed sample. The circumbinary gas was observed in [NII] 6584, which is the strongest line in most extended nebulae (Corradi et al. 1999b).

In this paper we focus on $\mathrm{He} 2-104$ which belongs to the 8 D-type SBN in the Corradi et al. sample. The image, which appeared in an HST News Release on August 1999 (http://oposite.stsci.edu/pubinfo/pr/1999/32/) is presented in Fig. 1 (see also Corradi 2000, Fig. 1).

The morphology of He2-104 is described by Corradi \& Schwarz (1993). The line spectra emitted by the inner nebula of He2-104 have been observed by Lutz et al. (1989) and Freitas Pacheco \& Costa (1996). The line ratios indicate a large range of temperatures and densities. Actually, Freitas Pacheco \& Costa "did not succeed in reproducing the observed line ratios" by pure photoionisation models,

Send offprint requests to: M. Contini, e-mail: contini@ccsg.tau.ac.il

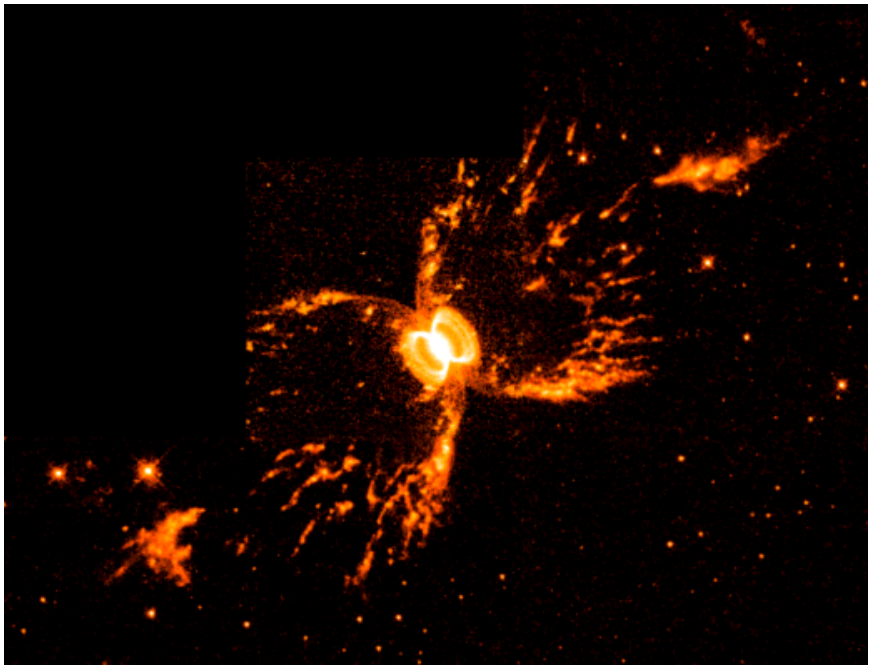

Fig. 1. HST [NII] image of He 2-104.

and suggested a contribution from shocks. Shocks were already invoked by Schwarz et al. (1989) for He2-104. In the present work we model the spectra by composite models which consistently account for the shock as well as for the radiation from the hot star. The SUMA code (Viegas \& Contini 1994) is adopted.

Narrow band images in [OIII] 5007, H $\alpha$, [NII] 6584, and [SII] 6731 were presented by Schwarz et al. (1989), Lutz et al. (1989), and Corradi \& Schwarz (1993). Detailed spectra of the outer nebula are not yet available, the only information being that $[\mathrm{NII}]$ is the brightest line (Corradi 2000). Nevertheless, the characteristics of the nebula are investigated on the basis of the qualitative information derived from the narrow-band images. We note that first 
ionisation level lines (e.g. [NII]) are generally strong in shock dominated spectra.

Composite models are presented in Sect. 2. He2-104 inner nebula spectra are modelled in Sect. 3 and the outer nebula is discussed in Sects. 4 and 5. Concluding remarks follow in Sect. 6.

\section{Composite models for the calculation of the spectra}

We have run a grid of composite models with the code SUMA (see Contini 1997). The input parameters are those referring to the hot star (the effective temperature, $T_{*}$, the ionisation parameter, $U$ ) and those which refer to the shock (the shock velocity, $V_{\mathrm{s}}$, the preshock density, $n_{0}$, and the preshock magnetic field, $\left.B_{0}\right)$. Moreover, the abundances relative to $\mathrm{H}$ of $\mathrm{He}, \mathrm{C}, \mathrm{N}, \mathrm{O}, \mathrm{Ne}, \mathrm{Mg}, \mathrm{Si}, \mathrm{S}, \mathrm{A}$, and $\mathrm{Fe}$, and the geometrical thickness, $D$, of the emitting filaments are also included.

Both Kaler (1976) and Kenyon \& Webbink (1984) suggest that for very hot stars the blackbody approximation provides a better fit to the nebular emission lines than the stellar models. A blackbody approximation is adopted in SUMA. Particularly, a grid of models with input parameters in the ranges suitable for $\mathrm{SBN}$ is presented by Formiggini et al. (1995, Table 3).

\section{The physical conditions in the inner nebula}

We apply to He2-104 the same model that was used for modelling SBN previously (Formiggini et al. 1995; Contini 1997; Contini \& Formiggini 1999), i.e. we assume that the spectrum is emitted from the interaction zone of two colliding stellar winds, one from the WD and the other from the late giant. Two shock fronts form at the collision (Contini \& Formiggini 2000), one propagating in reverse towards the hot star and the other expanding outwards of the system. The former is responsible for emission of lines from relatively high ionisation levels, the latter for low level and neutral lines.

The emitting nebulae are, therefore, heated and photoionised both by the shock created by collisions and by the radiation flux from the hot star. In the reverse shock both the radiation and the shock act on the internal edge of the emitting nebula. In the expanding shock they act on opposite edges.

We refer to the optical spectrum observed and reddening corrected by Freitas Pacheco \& Costa (1996). The calibration of the optical spectra observed by Lutz et al. (1989) is uncertain and the line fluxes are not reddeningcorrected, so they will be used only to further constrain the model. In Table 1 the strongest lines (relative to $\mathrm{H} \beta$ ) and the best fitting models are presented. The parameters are chosen phenomenologically by the fit of calculated to observed line ratios. Three reverse models (models 1 , 2 , and 3 ) and one expanding model (model $4^{*}$ ) are selected and the input parameters are listed at the bottom of Table $1 . B_{0}$ is $10^{-3}$ Gauss for all the models.
We refer to the observed CIV 1550/HeII 1640 line ratio by Lutz et al. to constrain the model. Both lines are strong and the frequencies close enough to depend less dramatically on the reddening correction, even in the UV. The observed ratio, $\sim 2$, is fitted only by model 2 which yields a value of 1.6 for it, whereas models 1 and 3 under-predict the ratios by a factor of 4 . Therefore, we adopt model 2 , characterized by $U=0.4$, as the best-fitting model. Notice that the reverse shock velocity of $300 \mathrm{~km} \mathrm{~s}^{-1}$ does not represent the velocity of the expanding matter observed by Schwarz et al. (1989), namely, $-36 \pm 18 \mathrm{kms}^{-1}$ in the northern lobe, $-139 \pm 12 \mathrm{~km} \mathrm{~s}^{-1}$ in the central bright region, and $-235 \pm 15 \mathrm{~km} \mathrm{~s}^{-1}$ in the southern lobe.

The calculated $[\mathrm{OII}] / \mathrm{H} \beta$ is, however, much smaller than the observed one in model 2 , as strong lines from low ionisation levels are generally emitted by the expanding shock (see e.g. Contini 1997 and Contini \& Formiggini 1999). This is represented by model $4^{*}$. The presence of two shocks is generally revealed by the line profiles (Contini 1997). Since we have no information from the data, we have adopted the expanding model presented by Contini \& Formiggini (1999, Table 3, model exp3) for the SBN RR Tel. The $\mathrm{H} \beta$ absolute flux is $386 \mathrm{erg} \mathrm{cm}^{-2} \mathrm{~s}^{-1}$ and $0.84 \mathrm{erg} \mathrm{cm}^{-2} \mathrm{~s}^{-1}$ for models 2 and $4^{*}$, respectively. The weighted sum of the spectra shows that the best fit is obtained by the weight ratio 1:10 for model 2 :model $4^{*}$. The results are given in the last column of Table 1.

$R_{\mathrm{OIII}} \equiv$ [OIII] $5007 /[\mathrm{OIII}] 4363$ is in good agreement with the observed value. $R_{\text {OIII }}$ depends strongly on the electron density and temperature of the emitting gas. High electron densities follow from compression downstream and high temperatures follow from the relatively high shock velocity.

The $[\mathrm{HeII}] / \mathrm{H} \beta$ line ratio, on the other hand, is determined particularly by $T_{*}$. The results of Table 1 indicate that the color temperature of the hot star is high enough $\left(T_{*}=130000 \mathrm{~K}\right)$ to unambiguously classify He2-104 as a symbiotic nova.

The relative abundances adopted in these calculations are $\mathrm{He} / \mathrm{H}=0.1, \mathrm{~N} / \mathrm{H}=1.5 \times 10^{-4}$, and $\mathrm{O} / \mathrm{H}=4.6 \times 10^{-4}$ (Formiggini et al. 1995).

The $[\mathrm{FeVI}] 5176 / \mathrm{H} \beta$ line ratio is under-predicted by model 2 but overpredicted by model 3 , while $[\mathrm{FeVII}] / \mathrm{H} \beta$ is rather well fitted. Therefore, a model between models 2 and 3 would better fit the $[\mathrm{FeVI}] / \mathrm{H} \beta$ line ratio. In this case the fit of all the line ratios would improve, except for CIV 1550/HeII 1640.

The [SII] 6716/6730 line ratio is under-predicted by model 2 , indicating higher electron densities in the emitting gas. Freitas Pacheco \& Costa find a density stratification from different line ratios, the density value deduced from the [SII] 6716/6730 ratio being the lower limit. A stratification of densities and temperatures downstream is characteristic of models which account for the shock. Moreover, the error of the observed lines is not quoted by Freitas Pacheco \& Costa. A line intensity error of $\sim 20 \%$ would improve the fit of the predicted ratio. 
Table 1. The comparison of calculated with observed line ratios to $\mathrm{H} \beta=1$.

\begin{tabular}{lllllll}
\hline line & obs $^{1}$ & model 1 & model 2 & model 3 & model $4^{*}$ & model SUM \\
\hline$[\mathrm{OII}] 3727$ & 0.057 & 0.036 & 0.01 & 0.021 & 1.2 & 0.04 \\
{$[\mathrm{NeIII}] 3869+$} & 1.9 & 2.27 & 2.77 & 2.38 & 2.5 & 2.72 \\
{$[\mathrm{OIII}] 4363$} & 0.72 & 0.38 & 0.83 & 0.52 & 0.16 & 0.8 \\
$\mathrm{HeII} 4686$ & 0.185 & 0.170 & 0.188 & 0.165 & 0.37 & 0.189 \\
{$[\mathrm{OIII}] 5007+$} & 4.0 & 4.16 & 4.16 & 4.24 & 17.2 & 4.38 \\
{$[\mathrm{FeVI}] 5176$} & 0.08 & 0.01 & 0.024 & 0.12 & - & - \\
$\mathrm{HeI} 5876$ & 0.14 & 0.13 & 0.13 & 0.124 & 0.13 & 0.13 \\
{$[\mathrm{FeVII}] 6086$} & 0.004 & 0.001 & 0.005 & 0.003 & - & - \\
{$[\mathrm{NII}] 6584+$} & 0.3 & 0.4 & 0.23 & 0.27 & 3.8 & 0.3 \\
{$[\mathrm{SII}] 6716$} & 0.0084 & 0.009 & 0.0084 & 0.008 & - & - \\
{$[\mathrm{SII}] 6730$} & 0.011 & 0.02 & 0.019 & 0.018 & - & - \\
\hline$V_{\mathrm{s}}\left(\mathrm{km} \mathrm{s}{ }^{-1}\right)$ & - & 150 & 300 & 300 & 50 & - \\
$n_{0}\left(10^{4} \mathrm{~cm}{ }^{-3}\right)$ & - & 7 & 7 & 5 & 2 & - \\
$T_{*}\left(10^{5} \mathrm{~K}\right)$ & - & 1.5 & 1.3 & 1.4 & 1.4 & - \\
$U$ & - & 0.05 & 0.4 & 0.15 & 0.005 & - \\
$D\left(10^{15} \mathrm{~cm}\right)$ & - & 0.67 & 4.47 & 8. & 2 & - \\
\hline
\end{tabular}

${ }^{1}$ Freitas Pacheco \& Costa (1996).

\section{The crab-like outer nebula}

In spite of the lack of quantitative data about the spectrum, some general considerations can be derived.

The emitting filaments (or the "crab legs", see Sect. 5) propagate outwards and a shock front develops on the outer edge. Radiation from the hot star reaches the inner edge of the filaments. The temperature of the hot star $\left(T_{*} \sim 130000 \mathrm{~K}\right)$ results from detailed modelling of the inner nebula (Sect. 3). An effective temperature of $T_{*}=130000 \mathrm{~K}$, implies an ionising photon flux of $\mathcal{N}=3.3 \times 10^{26}$ photons $\mathrm{cm}^{-2} \mathrm{~s}^{-1}$. This flux is related to the ionisation parameter, $U$, and to the gas (number) density downstream, $n$, in the radiation dominated region of the filaments, by: $\mathcal{N}\left(R_{\mathrm{WD}} / r\right)^{2}=U n c$, where $R_{\mathrm{WD}} \sim 10^{9} \mathrm{~cm}$ is the radius of the WD, and $r \sim 0.3 \mathrm{pc}$ (Corradi 2000) is the distance of the filament region from the hot source. We obtain:

$$
\begin{aligned}
U= & 0.01\left(T_{*} / 1.3 \times 10^{5} \mathrm{~K}\right)^{3} \\
& \times\left(R_{\mathrm{WD}} / 10^{9} \mathrm{~cm}\right)^{2}\left(r / 10^{18} \mathrm{~cm}\right)^{-2} / n .
\end{aligned}
$$

The downstream compression $\left(n / n_{0}\right)$ increases with higher $V_{\mathrm{s}}$ and decreases with higher $B_{0}$. Even adopting for $n_{0}$ the ISM density value $\left(n_{0} \geq 1 \mathrm{~cm}^{-3}\right)$ the resulting $n$ would lead to a very low $U$. This is reasonable in view of the large distance of the filaments from the hot source. The gas inside the filaments will be mainly ionised and heated by the shock. Consequently, shock dominated $(U=0)$ models will be adopted in the calculation of line intensities.
The models selected from the grid, which show the observed spectrum characteristics (prominent [NII] 6584 line, absolute and relative to [OIII] 5007) are presented in Table 2. The input parameters appear in Cols. 2-5, and in Cols. 6-9 the absolute fluxes of the [OIII] 5007, [OII] 3727, [NII] 6584, and $\mathrm{H} \beta$ lines are presented. The observed expansion velocities of the outer nebula are about $50-100 \mathrm{~km} \mathrm{~s}^{-1}$ (Corradi \& Schwarz 1993) and $\sim 250 \mathrm{~km} \mathrm{~s}^{-1}$ in the blobs related to the jets (Corradi \& Schwarz 1995). So, we present in Table 2 the results of calculations for $V_{\mathrm{s}}$ between 50 and $250 \mathrm{~km} \mathrm{~s}^{-1}$.

In Fig. 2 the physical conditions of the emitting gas relative to models $\mathrm{O} 1$ and $\mathrm{O} 6$ (Table 2), which show extreme $V_{\mathrm{s}}$, are presented.

The electron densities downstream in both diagrams (Fig. 2) are in agreement with the densities of 300$1000 \mathrm{~cm}^{-3}$ found in the "crab legs" by Corradi \& Schwarz (1993). Interestingly, for model O1 (Fig. 2 left) $\mathrm{N}^{+1}$ is lower than $\mathrm{O}^{+1}$, while $F_{[\mathrm{NII}]}$ is higher than $F_{[\mathrm{OII}]}$. In fact, both the ionisation potential, $\chi$, and the collision strength, $\Omega$, are important in the calculation of collisional excited lines: $F_{\text {line }} \propto \Omega \exp (-\chi) Y_{i} / Y$ (Osterbrock (1989). Actually, $\chi$ is $1.90 \mathrm{eV}$ and $3.32 \mathrm{eV}$ for the transitions ${ }^{3} \mathrm{P}-{ }^{1} \mathrm{D}(\mathrm{NII})$ and ${ }^{4} \mathrm{~S}-{ }^{2} \mathrm{D}(\mathrm{OII})$, respectively, and $\Omega$ is 2.99 and 1.47 , respectively, leading to the intensities shown in Table 2 .

The relative high $V_{\mathrm{s}}\left(250 \mathrm{~km} \mathrm{~s}^{-1}\right)$ in model 06 creates a large zone of high temperature gas $\left(\leq 10^{6} \mathrm{~K}\right)$, which corresponds to soft X-ray emission from the postshock region of the blobs. However, this model refers to the 
Table 2. The line fluxes calculated at the emitting nebula (in $\mathrm{erg} \mathrm{cm}^{-2} \mathrm{~s}^{-1}$ ).

\begin{tabular}{lllllllll}
\hline & $\begin{array}{l}V_{\mathrm{s}} \\
\left(\mathrm{km} \mathrm{s}^{-1}\right)\end{array}$ & $\begin{array}{l}n_{0} \\
\left(10^{4} \mathrm{~cm}^{-3}\right)\end{array}$ & $\begin{array}{l}B_{0} \\
\left(10^{-5} \mathrm{Gauss}\right)\end{array}$ & $\begin{array}{l}D \\
\left(10^{16} \mathrm{~cm}\right)\end{array}$ & $F_{[\mathrm{OIII}]}$ & $F_{[\mathrm{OII}]}$ & $F_{[\mathrm{NII}]}$ & $F_{\mathrm{H} \beta}$ \\
\hline model O1 & 50 & 1 & 1 & $>1$ & 0.00024 & 0.029 & 0.048 & 0.0046 \\
model O2 & 50 & 1 & 10 & 0.1 & 0.00024 & 0.029 & 0.047 & 0.0044 \\
model O3 & 50 & 2 & 1 & 1 & 0.0004 & 0.03 & 0.07 & 0.0093 \\
model O4 & 100 & 1 & 1 & 2.5 & 0.15 & 0.049 & 0.24 & 0.49 \\
model O5 & 150 & 0.1 & 1 & 1 & 0.0027 & 0.012 & 0.014 & 0.00167 \\
model O6 & 250 & 0.1 & 100 & $>1$ & 0.08 & 0.33 & 1.1 & 0.096 \\
\hline
\end{tabular}
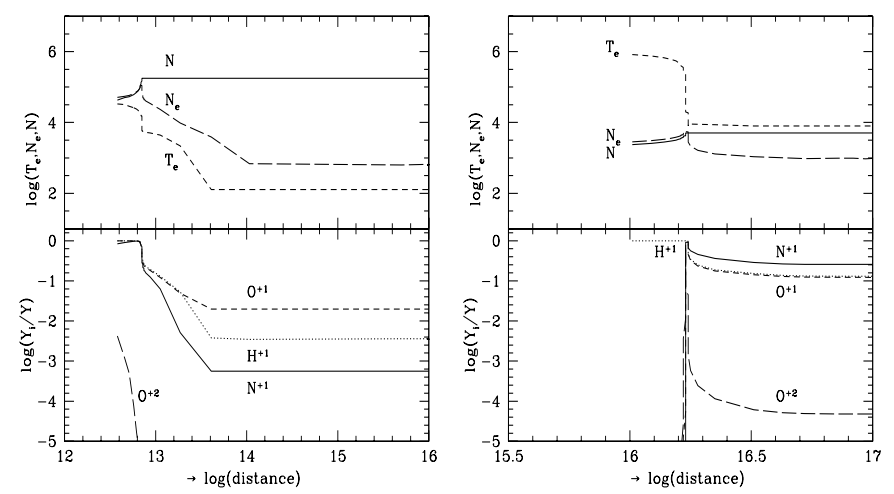

Fig. 2. Model O1 left, model O6 right. Top panels: The profiles of the electron temperature (short dashes), the electron density (long dashes), and of the density (solid line) as a function of distance from the shockfront (on the left side of each diagram). Bottom panels: The profiles of the fractional abundances of the most significant ions $\left(\mathrm{N}^{+1}\right.$ : solid line, $\mathrm{O}^{+1}$ : short-dashed line, $\mathrm{H}^{+1}$ : dotted line, $\mathrm{O}^{+2}$ : long-dashed line) as function of distance from the shockfront.

jets, whose angle is very small (see Fig. 1). The calculated X-ray flux is $0.036 \mathrm{erg} \mathrm{cm}^{-2} \mathrm{~s}^{-1}$ at the nebula and $\ll 10^{-13} \mathrm{erg} \mathrm{cm}^{-2} \mathrm{~s}^{-1}$ at earth, if the jet projected opening angle is $<5^{\circ}$. Since the angle is $\ll 5^{\circ}, \mathrm{He} 2-104$ flux is below the ROSAT all-sky- survey detection limit $\left(\sim 10^{-13} \mathrm{erg} \mathrm{cm}^{-2} \mathrm{~s}^{-1}\right)$.

\section{The "crab legs"}

The outer nebula filaments, named "crab-legs", are clearly identified in Fig. 1. The R-T instability created by turbulence at the shock front leads to fragmentation of matter. The strong resemblence between the "crab legs" which appear in the He2-104 image (Fig. 1) and the boundary of the shock front presented by Graham \& Zhang (2000) and, particularly, by Gull (1975, Fig. 3 ii) suggests that the "legs" are actually formed by R-T instability.

A rough evaluation of the filling factor can be derived from some general considerations. Adopting for He2-104 the typical Mira mass loss rate, $\dot{M}=10^{-6} M_{\odot} \mathrm{yr}^{-1}$, and an age $t=900$ yr (Corradi 2000), the ejected mass in
He2-104 is $M \sim 10^{-3} M_{\odot}$, The radius of the He2-104 outer nebula is about 0.3 pc (Corradi 2000). The swept-up mass to ejected mass ratio is about 7 , if the number density in the ISM is $2 \mathrm{~cm}^{-3}$. Assuming that all the ejected+swept up matter resides in the "crab legs", the filling factor, $f$, in the outer nebula can be calculated by $M(1+7)=$ $4 \pi r^{2} d d m_{\mathrm{H}} n_{\text {leg }} f$, where $d d$ is the geometrical thickness of the region containing most of the "legs". We roughly derive from the image in Fig. 1 that dd is of the order of $\sim 3.5 \times 10^{17} \mathrm{~cm}$. Adopting $M=2 \times 10^{30} \mathrm{~g}, r=10^{18} \mathrm{~cm}$, and a density in the "legs" $n_{\text {leg }}=1000 \mathrm{~cm}^{-3}$, the filling factor $f$ results $\sim 0.002$, in agreement with the clumpy structure of the outer nebula.

\section{Concluding remarks}

In this paper we have modelled the spectra emitted from both the inner and the outer nebulae around He2-104. The results of model calculations show the important role of the shocks. It is found that in the inner nebula the preshock density is $n_{0}=7 \times 10^{4} \mathrm{~cm}^{-3}$. In the outer nebula $n_{0}=10^{3}-10^{4} \mathrm{~cm}^{-3}$. The high gap between the density in the outer nebula "crab legs" and that in the ISM shows that the characteristics of the original ejected matter, even if partly washed out by merging with the ISM, are still recognizable. The ionisation parameter is $U=0.4$ in the inner nebula and negligible in the outer one, indicating that the effect of shocks dominates far away from the symbiotic system. Shock velocities of $50-250 \mathrm{~km} \mathrm{~s}^{-1}$ provide the prevailing [NII] line in the outer nebula. Soft X-ray emission is predicted from the jet blobs which show the highest shock velocity. The magnetic field is $B_{0}=10^{-3}$ Gauss in the inner nebula and $10^{-3}-10^{-5}$ Gauss in the outer nebula. Finally, the temperature of the hot star which results from modelling is $T_{*} \sim 130000 \mathrm{~K}$.

We suggest that the formation of the characteristic "crab legs" is due to R-T instability at the shock front.

More accurate and detailed results will be obtained by modelling when quantitative spectra rich in number of lines will be available from observations. 
Acknowledgements. We are grateful to D. Prialnik for precious comments, and to the referee, H. E. Schwarz, for helpful criticism.

\section{References}

Contini, M. 1997, ApJ, 483, 887

Contini, M., \& Formiggini, L. 1999, ApJ, 517, 925

Contini, M., \& Formiggini, L. 2000, in Thermal and Ionization aspects of the flows from hot stars: observations and theory, ed. Lamers, \& Sapar, ASP Conf. Ser., 204, 345

Corradi, R. L. M. 2000, in Thermal and Ionization aspects of the flows from hot stars: observations and theory, ed. Lamers, \& Sapar, ASP Conf. Ser., 204, 365

Corradi, R. L. M., \& Schwarz, H. E. 1993, A\&A, 268, 714

Corradi, R. L. M., \& Schwarz, H. E. 1995, A\&A, 293, 871
Corradi, R. L. M., Brandi, E., Ferrer, O. E., \& Schwarz, H. E. 1999a, A\&A, 343, 841

Corradi, R. L. M., Ferrer, O. E., Schwarz, H. E., Brandi, E., \& Garcia, L. 1999b, A\&A, 348, 978

Formiggini, L., Contini, M., \& Leibowitz, E. M. 1995, MNRAS, 277, 1071

Freitas Pacheco, J. A., \& Costa, R. D. D. 1996, A\&A, 309, 629

Graham, M. J., \& Zhang, Q. 2000, ApJS, 127, 339

Gull, S. F. 1975, MNRAS, 171, 263

Hack, W. J., \& Paresce, F. 1993, PASP, 105, 1273

Kaler, J. B. 1976, ApJ, 210, 843

Kenyon, S. J., \& Webbink, R. F. 1984, ApJ, 279, 252

Lutz, J. H., Kaler, J. B., Shaw, R. A., Schwarz, H. E., \& Aspin, C. 1989, PASP, 101, 966

Schwarz, H. E., Aspin, C., \& Lutz, J. H. 1989, ApJ, 344, L29

Viegas, S. M., \& Contini, M. 1994, ApJ, 428, 113 